\title{
Identification of serum biomarkers for colon cancer by proteomic analysis
}

\author{
DG Ward', N Suggett', , Y Cheng', W Wei', H Johnson', LJ Billingham', T Ismail ',2, MJO Wakelam', \\ PJ Johnson' and A Martin*,I \\ 'CR-UK Institute for Cancer Studies, University of Birmingham, Edgbaston, Birmingham B 5 2TT, UK; ${ }^{2}$ University Hospital Birmingham, Birmingham, UK
}

Colorectal cancer (CRC) is often diagnosed at a late stage with concomitant poor prognosis. Early detection greatly improves prognosis; however, the invasive, unpleasant and inconvenient nature of current diagnostic procedures limits their applicability. No serum-based test is currently of sufficient sensitivity or specificity for widespread use. In the best currently available blood test, carcinoembryonic antigen exhibits low sensitivity and specificity particularly in the setting of early disease. Hence, there is great need for new biomarkers for early detection of CRC. We have used surface-enhanced laser desorbtion/ionisation (SELDI) to investigate the serum proteome of $62 \mathrm{CRC}$ patients and 31 noncancer subjects. We have identified proteins (complement C3a des-arg, $\alpha \mathrm{l}$-antitrypsin and transferrin) with diagnostic potential. Artificial neural networks trained using only the intensities of the SELDI peaks corresponding to identified proteins were able to classify the patients used in this study with $95 \%$ sensitivity and $91 \%$ specificity. British Journal of Cancer (2006) 94, I898-1905. doi:I0.1038/sj.bjc.6603। 88 www.bjcancer.com

Published online 6 June 2006

(C) 2006 Cancer Research UK

Keywords: colorectal cancer; SELDI; serum proteome; biomarker; proteomic; mass spectrometry

Colorectal cancer (CRC) is a major cause of worldwide morbidity and mortality and is the second most common cause of cancer death in Europe and the United States causing more than 50000 deaths in the US and 16000 in the UK each year (Greenlee et al, 2001; CRUK, 2004). CRC follows a gradual progression from benign polyps through early cancers to late and metastatic cancers (Jackman and Mayo, 1951; Tierney et al, 1990). Screening programmes for early diagnoses have resulted in a reduction in mortality (Newcomb et al, 1992; Selby et al, 1992; Muller and Sonnenberg, 1995) because survival decreases with increasing stage. Endoscopic examination of the colon remains the gold standard for diagnosis; however, this is invasive, unpleasant and carries associated risk of morbidity and mortality. Identification of high-risk patients using a less invasive test would decrease the number of such procedures required. Carcinoembryonic antigen (CEA) is of proven benefit in prognosis and follow-up, but has limited sensitivity $(30-40 \%)$ for early CRC (Fletcher, 1986), whereas serial faecal occult blood testing is proven to reduce CRC mortality but suffers from significant false-negative and falsepositive rates (Hardcastle et al, 1989; Mandel et al, 1993; Kronberg et al, 1996). Stool DNA analysis for multiple targets has shown sensitivity of $71-91 \%$ in preliminary studies and larger studies are underway (Ahlquist et al, 2000; Dong et al, 2001); however, a serum-based assay with equivalent sensitivity and specificity would be more acceptable to many patients.

Surface-enhanced laser desorption/ionisation (SELDI) mass spectrometry (MS) is a technology that can produce proteomic

*Correspondence: Dr A Martin; E-mail: a.martin@bham.ac.uk Received 2 February 2006; revised 27 April 2006; accepted 27 April 2006; published online 6 June 2006 'fingerprints' from biological samples using a relatively highthroughput platform. The sample is diluted in an appropriate buffer and applied to 'proteinchip arrays' coated with chromatographic surfaces (anion and cation exchange, reverse-phase and immobilised divalent metal ion surfaces). A proportion of the peptides/proteins in the sample bind to the chip surface and the rest of the proteins and any other nonbinding components are rinsed away. Following addition of an energy absorbing organic acid the proteins on the surface are ionised into the gas phase using a laser and analysed by time-of-flight mass spectrometry (i.e. according to their mass/charge ratio). Multivariate analysis can then be used to determine whether the intensities of the peaks in the SELDI spectra of different patient groups possess discriminatory ability. Studies have suggested the possible utility of SELDI analysis in diagnosing ovarian (Petricoin et al, 2002; Kozak et al, 2003), prostate (Adam et al, 2002; Qu et al, 2002; Banez et al, 2003), breast (Li et al, 2002), bladder (Vlahou et al, 2001), hepatic (Poon et al, 2003; Ward et al, 2006) and pancreatic cancer using serum. Concerns with several aspects of this approach have been raised, including potential bias in sample collection protocols, accuracy/ resolution of the PBS IIc mass spectrometer employed in SELDI analysis, alignment of the detected peaks and over fitting of the data (Ransohoff, 2004). However, the potential benefits of a sensitive and reliable serum-based diagnostic test for a range of diseases, including cancer are so great that many efforts are being made to solve these problems.

More recently, SELDI and other MALDI-based approaches have been used to detect proteins that are differentially expressed between patient groups that can then be isolated and identified, often using MS/MS approaches (Le et al, 2005; Malik et al, 2005; Paradis et al, 2005). This information could be useful in the design of more specific diagnostic tests or inform us about the disease process. 
In this report, we describe the analysis of noncancer and CRC samples by SELDI and identify proteins responsible for peaks which characterise the CRC samples and therefore have the potential to function as biomarkers.

\section{MATERIALS AND METHODS}

\section{Patient/sample information}

Serum samples were obtained from patients attending the University Hospital Birmingham rapid-access clinic for primary care referrals with suspected CRC, or from healthy volunteers. Ethical approval was obtained for sample collection and all patients gave informed consent. Blood was collected into standard hospital blood collection tubes and allowed to clot at $4{ }^{\circ} \mathrm{C}$ for $1-2 \mathrm{~h}$, then warmed to room temperature for $30 \mathrm{~min}$ before centrifugation $(2500 \mathrm{~g}$ for $10 \mathrm{~min})$ and the serum aliquoted and stored at $-80^{\circ} \mathrm{C}$. Cancers $(62$ samples $)$ and controls (31 samples) were collected into identical tubes and processed in an identical manner.

The noncancer group consisted of 13 male and 18 female patients vs 36 male and 26 female patients for CRC, aged $62.9 \pm$ 10.3 years for the noncancer $v s 67.3 \pm 12.9$ years for CRC. The CRC group contained 27 patients with localised disease (Dukes' $\mathrm{A} / \mathrm{B}$ ) and 35 patients with disseminated disease (Dukes' C/D). The noncancer group (31 samples) contained patients from the same rapid-access clinic (27) as the CRC patients (12 diverticular disease, 15 no abnormality detected) or healthy volunteers (4). The noncancers were predominantly individuals referred to the rapidaccess clinic because of indicative symptoms that were determined to not have CRC and the cancers were individuals proven to have CRC by attending the same clinic. This represents the 'real-world' comparison presented to colon practitioners when diagnosing colon cancer, rather than healthy controls that may not constitute as relevant a comparison for a diagnostic test.

\section{SELDI analysis}

Sera were analysed on $\mathrm{Cu}^{2+}$-loaded IMAC30 proteinchip arrays. The samples (including duplicates) were randomised with respect to position in the bioprocessor. IMAC proteinchip arrays were prepared by incubation with $100 \mathrm{mM} \mathrm{CuSO}_{4}$ for $5 \mathrm{~min}(50 \mu \mathrm{l}$ per spot) followed by a water rinse and $3 \times 10$ min washes with $200 \mu \mathrm{l}$ binding buffer ( $\left.500 \mathrm{~mm} \mathrm{NaCl}, 100 \mathrm{~mm} \mathrm{NaH} \mathrm{PO}_{4} / \mathrm{NaOH}, \mathrm{pH} 7.0\right)$. All sera were diluted five-fold in $9 \mathrm{~m}$ urea, $50 \mathrm{~mm}$ Tris/ $\mathrm{HCl}, \mathrm{pH} 9.0$, $2 \%\left(\mathrm{w} \mathrm{v}^{-1}\right)$ CHAPS, followed by a 10 -fold dilution in binding buffer before the addition of $100 \mu$ l diluted sample per well. Binding was allowed to proceed for $1 \mathrm{~h}$ at room temperature with shaking at 900 r.p.m. The proteinchip arrays were then washed four times using $200 \mu \mathrm{l}$ of binding buffer ( $10 \mathrm{~min}$ with shaking) followed by a water rinse. The proteinchip arrays were allowed to dry and $1 \mu \mathrm{l}$ of a $50 \%$ saturated solution of sinapinic acid in $50 \%$ acetonitrile, $0.5 \%$ trifluoroacetic acid (matrix solution) applied to each spot. After air drying, another $1 \mu \mathrm{l}$ of matrix solution was added and the spots air-dried before analysis in a PBS IIc SELDI-TOF equipped with an autoloader (Ciphergen, Biosystems Inc., Fremont, CA, USA). Spectra were collected over 0-20 and $0-200 \mathrm{kDa}$ ranges (600 laser shots) using laser intensity settings of 165/185 (low range/high range). Spectra were externally calibrated using neurotensin, cytochrome $c$, myoglobin, chymotrypsinogen and bovine serum albumin (Sigma-Aldrich, Poole, Dorset, UK) and the intensities normalised using the total ion current. Spectra with a total ion current of less than $20 \%$ of the average for the experiment were excluded from the analysis. Peaks were detected automatically using Ciphergen proteinchip software (valley depth and peak height both set at two times the noise) and those peaks present in $>10 \%$ of the spectra clustered using the Biomarker Wizard tool (manufacturer's default settings). Peak intensities for duplicate spectra were combined and compared between the noncancer and CRC groups using twosample $t$-test and the area under the receiver operator characteristic (ROC) curve. Peaks found to be statistically significantly different between the groups were used to develop artificial neural networks (ANNs).

\section{Sample classification}

Artificial neural networks were used to classify serum samples into cancer and noncancer as described previously (Ward et al, 2006). The feedforward neural networks consisted of three layers: an input layer, a hidden layer and an output layer. The number of input nodes was determined by the number of significant peaks from which the models were trained. The hidden layer connected the input and output layers and the number of nodes in this layer controlled the complexity and performance of the neural networks. The output layer consisted of a single node whose output was used to classify sample status, representing cancer or noncancer. The model had full connection from the input nodes to the hidden nodes and from the hidden nodes to the output node. All of the connection weights were randomly initialised in the range $(-1$, $+1)$. The networks were trained using the back propagation algorithm and tested using 10 -fold cross validation.

\section{Biomarker purification}

The initial step in the purification of the $6.44,6.64,8.94$ and $50.7 \mathrm{kDa}$ peaks (based on a method validated by our group previously; Poon et al, 2003) was to dilute the serum $(100 \mu \mathrm{l})$ threefold in $9 \mathrm{~m}$ urea, $50 \mathrm{~mm}$ Tris/ $\mathrm{HCl}, \mathrm{pH} 9$ and $2 \%$ CHAPS buffer, and apply it to Q Ceramic HyperD F anion exchange beads (Pall, New York, USA) in spin-cup filters (Pierce, Rockford, Illinois, USA). The proteins that did not bind were collected by centrifugation.

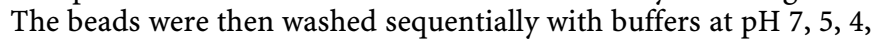
and 3 , and finally with $50 \%$ acetonitrile $+0.5 \%$ trifluoroacetic acid and the eluates collected. The $8.94 \mathrm{kDa}$ peak did not bind to the beads and the only additional purification step was SDS-PAGE. The 6.64 and $6.44 \mathrm{kDa}$ peaks bound to the beads and eluted predominantly at $\mathrm{pH}$. This fraction was applied to a $\mathrm{C}-18$ reverse-phase column (Vydac, $4.6 \times 300 \mathrm{~mm}$ ) equilibrated in solvent A ( $0.1 \%$ TFA in water) and proteins eluted using a linear gradient of $0 \%$ solvent B $(0.08 \%$ TFA in acetonitrile) to $100 \%$ B over $40 \mathrm{~min}$ at a flow rate of $0.5 \mathrm{ml} \mathrm{min}{ }^{-1}$. The fractions were analysed by SELDI and those containing the 6.64 and $6.44 \mathrm{kDa}$ peaks concentrated by vacuum evaporation and then separated by SDS-PAGE. The $50.7 \mathrm{kDa}$ peak eluted at $\mathrm{pH} 4$ and was also separated using reverse phase HPLC and analysed by SELDI but this time using the second dimension of a Beckman Coulter PF 2D protein purification system (see below).

The $79.1 \mathrm{kDa}$ protein was purified using a Beckman Coulter PF 2D-automated two-dimensional chromatography system. Pooled noncancer and pooled CRC sera were run in triplicate on the system. The sera were diluted in the $\mathrm{pH} 8.5$ 'start buffer' and the protein concentration measured (Pierce BCA protein assay). Total protein $(2.5 \mathrm{mg})$ for each sample was applied separately to the first dimension (chromatofocusing) at a flow rate of $0.2 \mathrm{ml} \mathrm{min}^{-1}$, a $\mathrm{pH}$ gradient formed using 'elution buffer' ( $\mathrm{pH} 4.0$ ) and fractions collected every $0.3 \mathrm{pH}$ unit. Fractions from this first dimension were diluted and applied to $\mathrm{Cu}^{2+}$-loaded IMAC proteinchip arrays and the fractions containing the $79.1 \mathrm{kDa}$ peak determined. The second dimension consists of a monolithic C-18 reverse phase column (equilibrated in solvent A) used to fractionate sequentially each of the first dimension fractions. Proteins were eluted using a linear gradient of solvents A to B over $30 \mathrm{~min}$ at a flow rate of $0.75 \mathrm{ml} \mathrm{min}^{-1}$ and fractions collected. Proteins eluted from the second dimension separation were 
quantified by measuring the absorbance at $214 \mathrm{~nm}$. The second dimension fractions derived from the first dimension fractions that contained the $79.1 \mathrm{kDa}$ peak were again screened by SELDI and the fractions containing the $79.1 \mathrm{kDa}$ peak were separated by SDS - PAGE.

The individual proteins from the purification schemes given above were processed for liquid chromatography-tandem mass spectrometry (LC-MS/MS). Briefly, the band of interest was excised, washed, reduced using $50 \mathrm{~mm}$ dithiothreitol, alkylated with $100 \mathrm{~mm}$ iodoacetamide and digested overnight with $250 \mathrm{ng}$ modified trypsin (Promega, Madison, Wisconsin, USA). The LC-MS/MS analysis was performed using an LC Packings Ultimate HPLC system linked to a ThermoFinnigan LCQ Deca XP Plus ion-trap mass spectrometer via a nanospray interface fitted with a metal emitter tip. The peptides were separated using a $180 \mu \mathrm{M}$ ID ThermoFinnigan BioBasic C-18 reverse phase-column run at $1.25 \mu \mathrm{min}^{-1}$ that was equilibrated with $95 \%$ solvent C $(5 \%$ acetonitrile in water $/ 0.1 \%$ formic acid) and $5 \%$ solvent D (95\% acetonitrile in water $/ 0.1 \%$ formic acid) and eluted with a gradient of $5-37.5 \% \mathrm{D}$ over $25 \mathrm{~min}$. The ion-trap was set to detect positively charged ions using a spray voltage of $2.5 \mathrm{kV}$ and an automated data-dependent MS/MS analysis performed on the five most abundant ion species from each MS full scan before another MS full scan was performed. Peptides were analysed a maximum of two times and were then placed on an exclusion list for $1 \mathrm{~min}$. The MS/MS spectra were searched against an NCBI nonredundant human database using TurboSequest as part of the Bioworks 3.1 suite of programmes. All analyses were carried out at least twice and only proteins with multiple peptides detected, using XCorr cutoff values of 2.5 for triply, 2.0 for doubly and 1.5 for singly charged ions, are given.

Western blots were performed on samples after SDS-PAGE using Immobilon PVDF membrane. The antibodies used were, anti-C3a antibody from Research Diagnostics Inc., Concorde, Massachusetts (catalogue number RDI-PRO61018), anti-apolipoprotein $\mathrm{C} 1$ from Chemicon International Inc., Temecula California (catalogue number MAB 1064), anti- $\alpha 1$-antitrypsin from Abcam, Cambridgeshire, UK (catalogue number ab9399) and antitransferrin from Abcam (ab1223). Where immunodepletions were performed the antibodies were pre-bound to Protein-G beads before addition to the serum. The depleted sera were retained following removal of the beads and associated proteins. The beads were washed and eluted with $50 \%$ acetonitrile/ $0.5 \%$ trifluoacetic acid. Control incubations using irrelevant antibodies or no antibody were performed to confirm specificity.

\section{Immunoassays}

The C3a ELISA was carried out using a kit from Research Diagnostics Inc. according to the manufacturer's instructions. Carcinoembryonic antigen was measured using a Roche Modular Immunoassay E170 analyser using the manufacturer's reagents and recommended methodology at the Worcestershire Acute Hospitals Trust.

\section{RESULTS}

A SELDI analysis of CRC patient's sera for the low range $(0-$ $20000 \mathrm{~m} / z)$ and high range $(0-200000 \mathrm{~m} / z)$ were performed and two-sample $t$-tests were carried out to determine which peak intensities were significantly different in the noncancer $v s$ CRC groups (see Table 1) using the SELDI spectra from individual patients. Varying numbers of the most significant peaks were then used to develop ANNs to discriminate between cancer and noncancer with 10-fold cross-validation. The ANNs developed using the seven most significant peaks performed best giving a sensitivity of $94 \%$ and specificity of $96 \%$.
A pooled CRC sample (containing serum from 46 individuals) and a pooled noncancer sample (26 individuals) were analysed in quadruplicate on 10 IMAC proteinchip arrays prepared at intervals over an 11-week period. This experiment was designed to assess the reproducibility of the analysis and also to confirm proteomic features characteristic of colon cancer. The average intrachip coefficient of variation (CV) for the peak intensities in both samples was $18 \%$ and the average CV for both samples across all 10 proteinchip arrays was $25 \%$. In addition, the Euclidean distance and correlation coefficient (always $>0.97$ ) between the peak heights on each of the 10 proteinchip arrays, relative to the first proteinchip array, did not show any trends across the experiment. These data demonstrate that SELDI spectra are reproducible over extended periods if materials and methods are not changed. The results in Table 2 show the peaks that are detected as significantly different between the two pooled samples (here the $P$-values do not reflect biological but simply experimental variation). Many of these peaks are the same (marked with ${ }^{*}$ ) as those found to be significantly different in the analysis of the individual samples

Table I Significant proteomic features from individual serum samples

\begin{tabular}{lccc}
\hline Peak (m/z) & $P$ (t-test) & AUC & Fold change \\
\hline 4790 & $6.0 \times 10^{-6}$ & 0.786 & 0.67 \\
50700 & $7.1 \times 10^{-6}$ & 0.798 & 1.71 \\
8940 & 0.00020 & 0.739 & 1.48 \\
6440 & 0.00026 & 0.705 & 0.68 \\
6640 & 0.00057 & 0.690 & 0.72 \\
123000 & 0.00065 & 0.712 & 0.75 \\
4290 & 0.00077 & 0.701 & 0.67 \\
8150 & 0.0014 & 0.682 & 1.31 \\
76000 & 0.0024 & 0.678 & 1.37 \\
8760 & 0.0035 & 0.721 & 0.62 \\
4480 & 0.0039 & 0.685 & 1.55 \\
79100 & 0.0043 & 0.676 & 1.21 \\
39900 & 0.0052 & 0.738 & 1.38 \\
\hline
\end{tabular}

AUC, area under the ROC curve. SELDI peaks significantly different in the sera of CRC patients. Serum samples from control and cancer patients were analysed in duplicate using $\mathrm{Cu}^{2+}$-loaded IMAC proteinchip arrays. The peak intensities between controls and cancer were compared and the fold change (cancer relative to controls) and significance are given. ROC curves for the significant peaks $(P>0.05)$ were constructed and the area under the curve for each peak is shown.

Table 2 Significant peaks from the analysis of pooled samples

\begin{tabular}{lcc}
\hline Peak (m/z) & $P(t$-test) & Fold change \\
\hline $8150 *$ & $1.4 \times 10^{-12}$ & 1.21 \\
$39900 *$ & $8.9 \times 10^{-12}$ & 1.56 \\
$79100 *$ & $1.0 \times 10^{-9}$ & 1.32 \\
$50700 *$ & $3.9 \times 10^{-9}$ & 1.31 \\
11530 & $1.0 \times 10^{-7}$ & 3.01 \\
9000 & $4.3 \times 10^{-7}$ & 1.22 \\
11690 & $1.3 \times 10^{-6}$ & 2.20 \\
2285 & $6.9 \times 10^{-6}$ & 0.72 \\
$4290 *$ & $1.8 \times 10^{-5}$ & 0.75 \\
5920 & $2.3 \times 10^{-5}$ & 0.87 \\
$8940 *$ & 0.00028 & 1.20 \\
7940 & 0.00039 & 1.09 \\
$4480 *$ & 0.00050 & 1.19 \\
$6640 *$ & 0.00076 & 0.79 \\
3970 & 0.0012 & 1.20 \\
$6440 *$ & 0.0016 & 0.78 \\
\hline
\end{tabular}

Pooled control and cancer samples were analysed 40 times using $\mathrm{Cu}^{2+}$-loaded IMAC proteinchip array. The peak intensities for the samples were compared and the significantly different peaks $(P>0.05)$ are listed along with the $P$-value and fold change. Peaks marked with * are those that are also significantly different in the SELDI profiles of the individual samples given in Table I. 
(Table 1). The significant proteomic features common to both experiments were considered as suitable candidates for purification and identification.

The initial strategy to isolate the peaks of interest was based on the serum fractionation protocol used by Poon et al (2003). The sera were diluted in a buffer designed to disrupt protein/protein interactions ( $9 \mathrm{~m}$ urea and 2\% CHAPS, $\mathrm{pH}$ 9). Under these conditions, proteins with a $\mathrm{pI}$ below $\mathrm{pH} 9$ bind to anion exchange resin and those with a $p \mathrm{I}$ above $\mathrm{pH} 9$ do not. The bound proteins can then be eluted by washing the resin sequentially with buffers of decreasing $\mathrm{pH}$. The nonbinding sample and the various eluates were analysed using $\mathrm{Cu}^{2+}$-loaded IMAC30 proteinchip arrays and the fractions containing the peaks of interest determined.

The $8.94 \mathrm{kDa}$ peak did not bind to the anion exchange resin at $\mathrm{pH} 9$, and when the nonbound material was separated by SDSPAGE, a differentially expressed band of approximately the expected mobility was seen (data not shown). In-gel digestion and LC-MS/MS analysis of this band detected four tryptic peptides (Table 3) derived from complement $\mathrm{C} 3$, a protein with a predicted mass of $185 \mathrm{kDa}$ that is cleaved to produce complement C3a with a mass of $9095 \mathrm{Da}$. This protein has its C-terminal arginine residue removed to produce complement $\mathrm{C} 3 \mathrm{a}$ des-arg with a mass of $8938 \mathrm{Da}$ corresponding closely to the mass of the differentially detected peak in the SELDI analysis. All of the complement C3 tryptic peptides detected were from within the complement C3a des-arg sequence and represent $27 \%$ of the C3a des-arg. Complement C3a des-arg has a predicted pI of 9.3 in agreement with the observation that this molecule did not bind to the anion exchange resin at $\mathrm{pH} 9$. The identity of the SELDI peak at $8.94 \mathrm{kDa}$ was verified using an anti-complement C3a antibody to deplete complement C3a from serum. The SELDI peak at $8.94 \mathrm{kDa}$ was specifically removed from the serum by the antibody treatment and was subsequently recovered following elution from the antibody (Figure 1) confirming the identity of the $8.94 \mathrm{kDa}$ peak as complement $\mathrm{C} 3 \mathrm{a}$ des-arg.

In order to relate the SELDI peak intensities with complement C3a des-arg abundance, a complement C3a des-arg ELISA was performed. The result in Figure 2 shows a correlation between the SELDI peak intensity and the ELISA determined C3a abundance.

The 6.44 and $6.64 \mathrm{kDa}$ peaks coeluted from the ceramic HyperD $\mathrm{F}$ anion exchange resin in the $\mathrm{pH} \mathrm{7-4} \mathrm{fractions.} \mathrm{The} \mathrm{sample}$ containing the most intense peaks ( $\mathrm{pH} 5$ elution) was separated using RP-HPLC and the eluted fractions screened by SELDI. The fractions containing the 6.44 and $6.64 \mathrm{kDa}$ peaks were separated using SDS-PAGE and a band with the correct mobility excised, digested and analysed using LC-MS/MS. Six peptides covering $56 \%$

Table 3 Tryptic peptides used to identify the $6.44 / 6.64$ and $8.94 \mathrm{kDa}$ biomarkers

\begin{tabular}{|c|c|c|}
\hline Peptide & $\mathbf{M H +}$ & ID \\
\hline FISLGEACK & 1025.2 & Complement $\mathrm{C} 3$ a residues $42-50$ \\
\hline FISLGEACKK & II53.4 & Complement $\mathrm{C} 3$ a residues $42-51$ \\
\hline VFLDCCNYITELR & 1703.9 & Complement $\mathrm{C} 3$ a residues $52-64$ \\
\hline KVFLDCCNYITELR & | 832.1 & Complement $\mathrm{C} 3$ a residues $51-64$ \\
\hline TPDVSSALDK & 1033.1 & Apolipoprotein $\mathrm{Cl}$ residues $1-10$ \\
\hline EFGNTLEDK & 1053.1 & Apolipoprotein $\mathrm{Cl}$ residues $|3-2|$ \\
\hline EWFSETFQK & 1202.3 & Apolipoprotein $\mathrm{Cl}$ residues $40-48$ \\
\hline TPDVSSALDKLK & 1274.5 & Apolipoprotein $\mathrm{Cl}$ residues $\mathrm{I}-12$ \\
\hline LKEFGNTLEDK & 1294.4 & Apolipoprotein $\mathrm{Cl}$ residues ||$-2 \mid$ \\
\hline MREWFESTFQK & 1489.7 & Apolipoprotein $\mathrm{Cl}$ residues $38-48$ \\
\hline
\end{tabular}

Partially purified proteins were separated using SDS-PAGE and the relevant gel slice excised, reduced, alkylated and trypsinised. The peptides were collected and subjected to LC-MS/MS analysis followed by a database search to identify the peptides. The upper panel shows the peptides derived from complement C3a and the lower panel the peptides from apolipoprotein $\mathrm{Cl}$. of the sequence of apolipoprotein $\mathrm{C} 1$ were detected (Table 3). Apolipoprotein $\mathrm{C} 1$ has a predicted sequence mass of $6631 \mathrm{Da}$ and an additional truncated form with threonine and proline removed from the N-terminus with a mass of $6433 \mathrm{Da}$ has been reported (Bondarenko et al, 1999). Immunodepletion of serum using an anti-apolipoprotein $\mathrm{C} 1$ antibody removed both the 6.44 and $6.64 \mathrm{kDa}$ peaks, which were recovered following elution from the antibody (Figure 3A). Western blot analysis of serum samples (Figure 3B) did not detect any differences in apolipoprotein $\mathrm{C1}$ concentration.

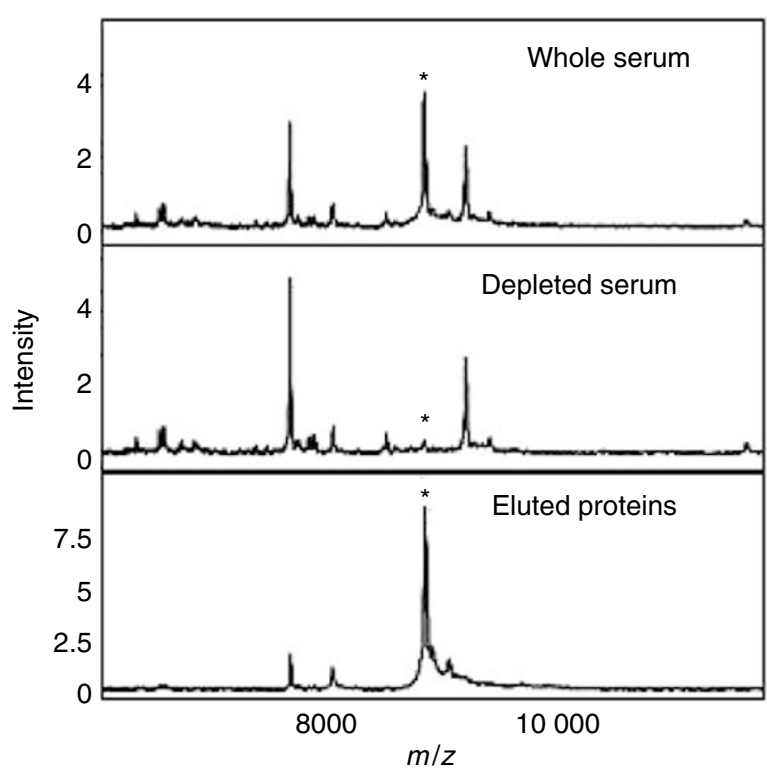

Figure I Immunodepletion of complement C3a des-arg. Serum was incubated with an anti-complement $\mathrm{C} 3$ a des-arg mouse monoclonal antibody bound to protein $\mathrm{G}$ sepharose. The protein $\mathrm{G}$ sepharose was collected by centrifugation and the non-bound proteins (depleted serum) retained. The beads were washed and the bound proteins eluted. The starting serum (upper panel), non-bound proteins (middle panel) and eluted proteins (lower panel) were analysed using $\mathrm{Cu}^{2+}$-loaded IMAC proteinchip arrays.

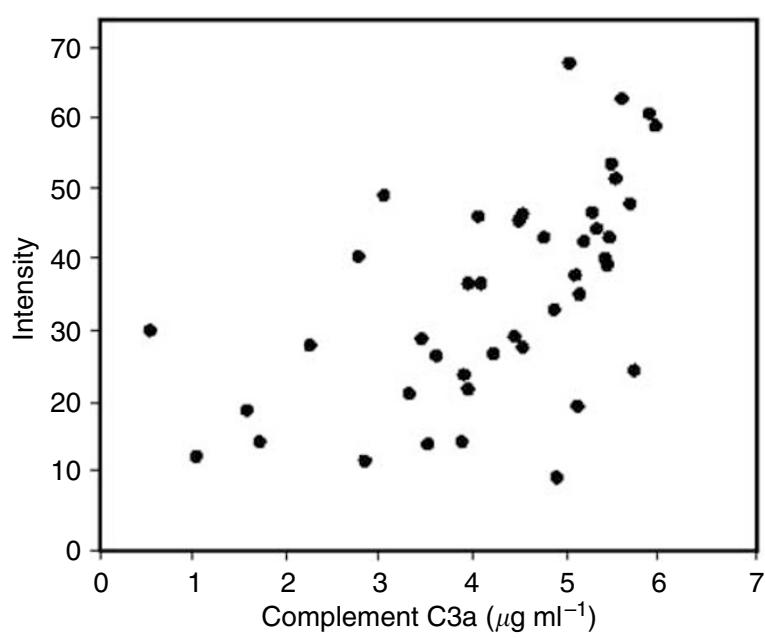

Figure 2 Comparison of the SELDI peak intensity at $8940 \mathrm{~m} / \mathrm{z}$ and the complement C3a levels in serum. The complement C3a des-arg level was measured using an ELISA kit from Research Diagnostics Inc. using the manufacturer's instructions. The results shown are the concentration of $\mathrm{C} 3 \mathrm{a}\left(\mu \mathrm{g} \mathrm{ml}^{-1}\right)$ plotted against SELDI peak intensity in the same sample. 

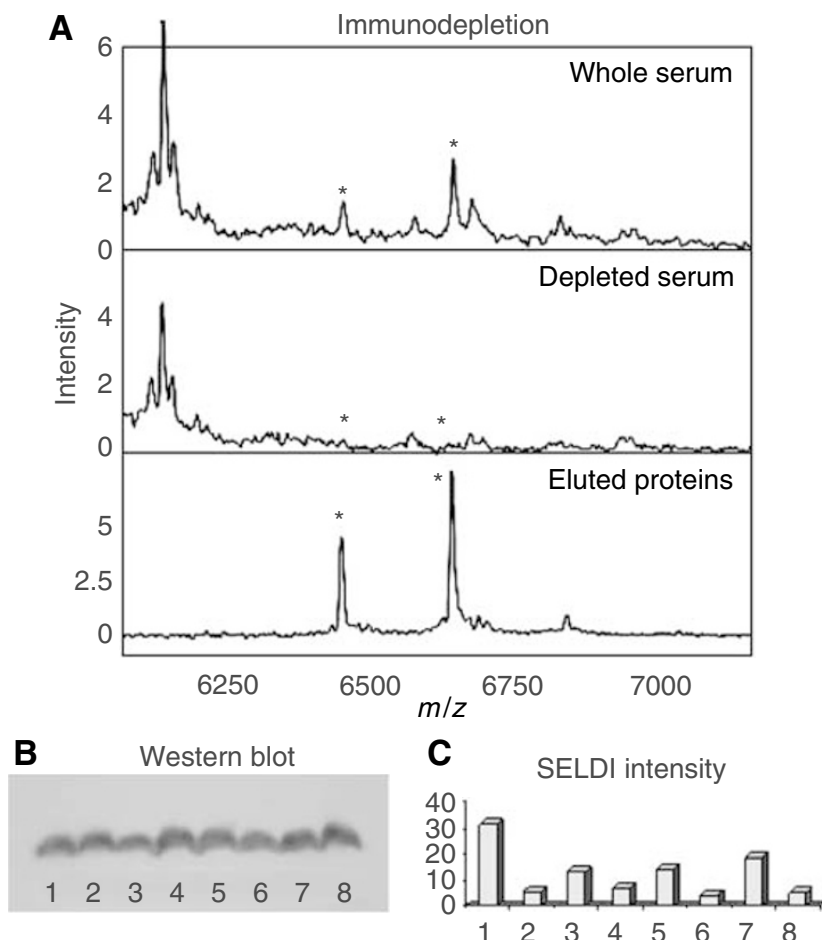

Figure 3 Immunodepletion of apolipoprotein $\mathrm{Cl}$ and a comparison of the intensity of the peak at $6640 \mathrm{~m} / \mathrm{z}$ with Western blot analysis of apolipoprotein $\mathrm{Cl}$. (A) Serum was depleted using an anti-apolipoprotein $\mathrm{Cl}$ mouse monoclonal antibody using the same protocol given for the immunodepletion of complement C3a given in Figure I. (B) A Western blot using an anti-apolipoprotein $\mathrm{Cl}$ antibody. The whole length and truncated forms of apolipoprotein $\mathrm{Cl}$ differ in mass by 198 Da and are not resolved by the SDS-PAGE so only a single band is observed. The samples were selected on the basis of a high or low SELDI peak height, as shown in (C). The SELDI peak intensity at $6640 \mathrm{~m} / \mathrm{z}$ using $\mathrm{Cu}^{2+}$-loaded IMAC proteinchip arrays for the same samples for the Western blot is shown. The $6440 \mathrm{~m} / \mathrm{z}$ peak displayed a similar pattern of intensities as the $6640 \mathrm{~m} / \mathrm{z}$ peak (results not shown)

The $50.7 \mathrm{kDa}$ biomarker eluted from the ceramic HyperD $\mathrm{F}$ anion exchange resin at $\mathrm{pH} 4$ and was further purified by RP-HPLC and the relevant fractions separated by SDS-PAGE. A band migrating with an apparent MW of $50.7 \mathrm{kDa}$ was excised and trypsinised, and the peptides harvested. LC-MS/MS analysis detected 27 unique peptides from $\alpha 1$-antitrypsin $(50 \%$ sequence coverage) and seven unique peptides from $\alpha 1$-antichymotrypsin (20\% sequence coverage). Both of these proteins are glycosylated, have similar molecular masses and isoelectric points and their co-purification has been reported previously (Koomen et al, 2005). It seemed likely that $\alpha 1$-antitrypsin is the major contributor to the $50.7 \mathrm{kDa}$ biomarker peak and this was confirmed by Western blot and immunodepletion. Furthermore, the SELDI peak intensity and Western blot staining (Figure 4) correlate, indicating that the concentration of $\alpha 1$-antitrypsin is higher in the serum of CRC patients in this study, as detected by SELDI peak height.

The $79.1 \mathrm{kDa}$ biomarker was purified by 2D liquid chromatography (Beckman-Coulter PF 2D system) resulting in an essentially pure $79.1 \mathrm{kDa}$ protein that binds to the IMAC proteinchip array (Figure 5B). This protein was identified as transferrin (44 unique peptides giving $57 \%$ sequence coverage). Absorbance at $214 \mathrm{~nm}$ of the transferrin revealed a modest increase in concentration in the pooled CRC sample relative to the pooled normal sample in agreement with the SELDI analysis, and Figure 5A shows the SELDI analysis of the immunodepletion/elution. The antitransferrin antibody decreased the intensity of the $79.1 \mathrm{kDa}$ peak, which was specifically detected in the eluted material.
A

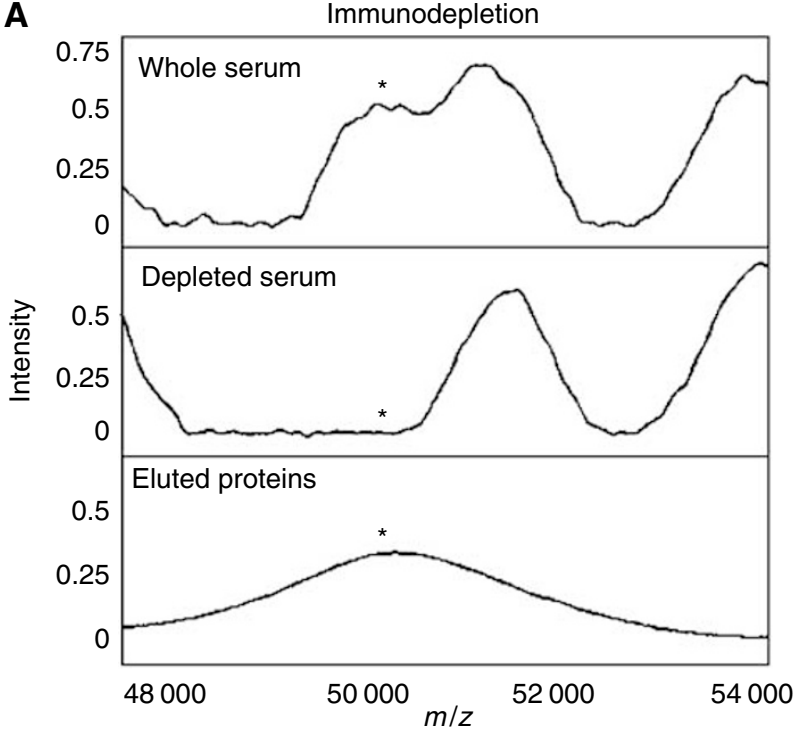

B

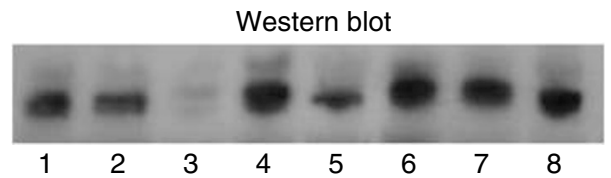

C

SELDI intensity

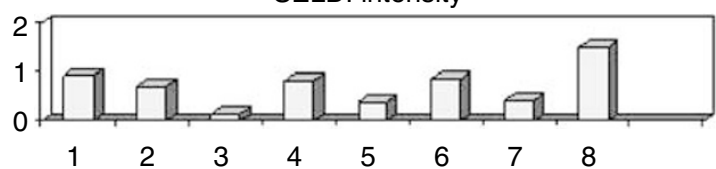

Figure 4 Immunodepletion of $\alpha$-antitrypsin and a comparison of the intensity of the peak at $50700 \mathrm{~m} / z$ with Western blot analysis of $\alpha \mathrm{l}$ antitrypsin. (A) An immunodepletion of $\alpha$-antitrypsin using a mouse monoclonal antibody was performed using the same strategy given in Figure I for complement C3a. (B) Western blot analysis of eight samples (C) Corresponding SELDI intensity for the $50700 \mathrm{~m} / \mathrm{z}$ peak. The samples used were selected on the basis of the $50700 \mathrm{~m} / \mathrm{z}$ peak intensity to asses the correlation between Western blot analysis and SELDI peak hight.

The SELDI spectra obtained during the purification of the transferrin showed a co-purifying peak of $39900 \mathrm{~m} / \mathrm{z}$ that is not seen in the stained gel (Figure $5 \mathrm{~B}$ and $\mathrm{C}$ ), which corresponds to the $\mathrm{m} / \mathrm{z}$ of a differentially expressed peak in the SELDI IMAC chip analysis of individual and pooled samples (Tables 1 and 2). When the transferrin immunodepletion was performed, the SELDI peak at $39.9 \mathrm{kDa}$ was also decreased and recovered in the eluted material (not shown) and commercially available purified transferrin displayed two peaks of approximately 79.1 and $39.9 \mathrm{kDa}$ (not shown). It is possible therefore that the $39.9 \mathrm{kDa}$ peak is the doubly charged transferrin ion, but the mass of the ion does not correspond accurately to the predicted $\mathrm{m} / z$ value, which may be due to the relatively low mass accuracy of the PBS II analyser.

\section{Sample classification using only the six SELDI peaks corresponding to apolipoprotein $\mathrm{C} 1$, complement C3a des-arg, $\alpha 1$-antitrypsin and transferrin}

Using unidentified peaks in SELDI spectra to classify patients is essentially a 'black box' approach. Having identified six of the peaks (the $6.44,6.64,8.94,50.7,79.1$ and the potentially doubly charged version of the $79.1 \mathrm{kDa}$ protein at $39.9 \mathrm{kDa}$ ), an ANN was developed using only these peaks. This ANN was able 
A

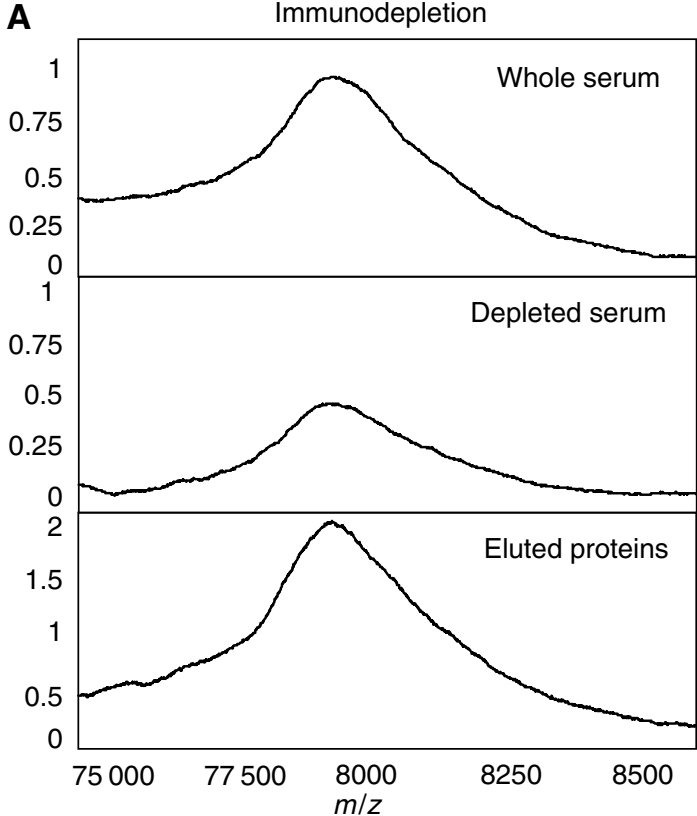

B

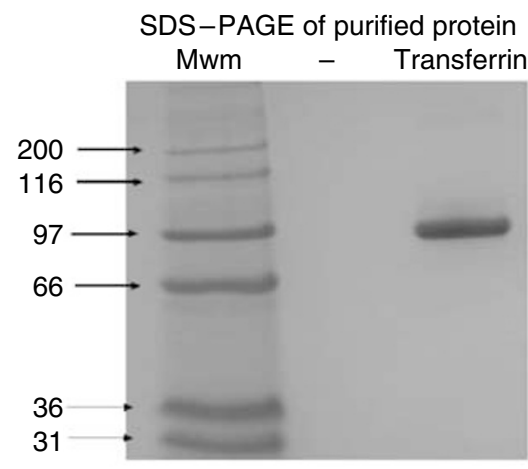

C

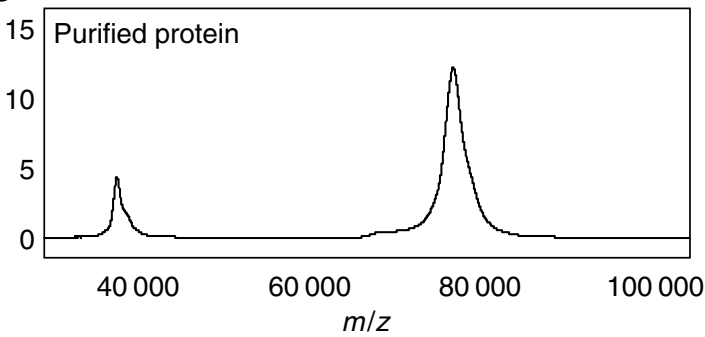

Figure 5 Immunodepletion and purification of transferrin. (A) An immunodepletion of transferrin employing an identical protocol to that in Figure I. The peak of $79100 \mathrm{~m} / z$ was purified by automated 2D HPLC and the fractions monitored using SELDI. A Coomassie-stained SDS-PAGE gel shows a clear single band at approximately $80 \mathrm{kDa}$ (B). (C) SELDI spectrum of this purified protein with a peak at the predicted size of $79100 \mathrm{~m} / \mathrm{z}$ in addition to an another at $39900 \mathrm{~m} / \mathrm{z}$.

to classify the samples with $95 \%$ sensitivity and $91 \%$ specificity (10-fold cross validation).

\section{Comparison and combination of ANNs with CEA}

Carcinoembryonic antigen was measured in all of the samples, and using the manufacture's recommended cutoff level of $4 \mathrm{ng} \mathrm{ml}^{-1}$, the sensitivity and specificity obtained was 53 and $93 \%$, respectively. Furthermore, inclusion of the CEA data in an ANN with the SELDI data for the six SELDI peaks identified here did not improve the ANN for the SELDI peaks alone.

\section{DISCUSSION}

The results presented in this paper demonstrate that SELDI analysis of CRC serum, compared to noncancer detects an altered intensity in a number of characteristic peaks which, when analysed by ANNs, have sensitivities and specificities in excess of $90 \%$. This work identified some of these peaks as transferrin, $\alpha 1$-antitrypsin, complement C3a des-arg and apolipoprotein C1. A similar use of SELDI by Chen et al (2004), Guang et al (2004) and Yu et al (2004) suggested that SELDI profiling could be more sensitive than CEA analysis in diagnosing CRC. The data in this paper support this, but, additionally, identifies potential biomarkers, which require validation with large numbers of patients and if successful could point to the development of more widely applicable immunoassays.

In this study, we screened for biomarkers in two distinct ways: (1) multiple measurements of two pooled samples containing serum from many noncancer controls or CRC patients and (2) duplicate measurements of serum from many noncancer individuals or patients with CRC. The first approach gave a thorough investigation of how reproducible SELDI peak heights were during this study: we find the intrachip CV to be within the manufacturer's specification and an overall CV of $25 \%$. Using the second approach, we collected duplicate SELDI spectra for each serum sample from 31 noncancer controls and 62 CRC patients. Having separate data on all the individuals in the study allowed us to assess diagnostic utility (area under the ROC curve, see Table 1) and to develop algorithms for patient classification. It can be seen that regardless of which of these two methods is employed, the majority of the peaks identified as significantly different in the CRC patients were the same (Tables 1 and 2).

The four proteins that we have identified as underlying six of the SELDI peaks with diagnostic potential are all classical serum proteins; however, this need not exclude their use as cancer biomarkers (Poon et al, 2001). The pair of peaks detected at 6440 and $6640 \mathrm{~m} / z$ were shown to be full-length apolipoprotein $\mathrm{C} 1$ and a truncated version that has been described previously (Bondarenko et al, 1999). Both of the peaks at 6440 and $6640 \mathrm{~m} / z$ were detected at decreased intensities in the CRC samples when the IMAC proteinchip array was employed (Tables 1 and 2). However, when the H50, Q10 and CM10 proteinchip arrays were used no differences in intensities were detected (results not shown). Furthermore, a Western blot for apolipoprotein $\mathrm{C} 1$ did not detect any difference between samples selected (Figure 3 ) on the basis of the intensity of the 6440 and $6640 \mathrm{~m} / z$ peaks determined using the IMAC proteinchip array. The reason for this is not clear but may be related to competition for binding at the retentate chromatography step and/or suppression of ionisation during the ionisation/desorption step. Presumably, the binding of these proteins to the IMAC proteinchip array and/or the ionisation/desorption step is influenced by underlying biochemical changes in one of the sample groups that do not interfere with the other proteinchip array types. This need not exclude the use of these discriminatory peaks in the development of ANNs to diagnose cancer if the observed differences are suitable for the purpose.

The identification of the peak at $8940 \mathrm{~m} / z$, as complement C3a des-arg using MS/MS analysis was confirmed using an immunodepletion approach (Figure 1). Chen et al (2004) also detected an elevation of a peak at $8930 \mathrm{~m} / z$ in samples from colon cancer patients that may be the same protein. A peak of similar mass was identified as apolipoprotein A-II in prostate cancer samples (Malik et al, 2005) and as a fragment of vitronectin in hepatocellular carcinoma samples (Paradis et al, 2005), underlining the need to validate identifications using independent assays. The increased 
level of complement C3a des-arg seen in the CRC patient sera suggested an increased level of complement activation indicative of inflammation. Complement C3a is highly biologically active, binding to mast cells and basophils and triggering release of their vasoactive contents (the des-arg form represents a stable inactivated form of complement C3a). The elevated level of complement C3a des-arg in the serum of CRC patients may reflect an immune response to the tumour, or possibly in vitro complement activation (Mollnes et al, 1988). This is unlikely to be a problem in this study as all samples were handled in an identical manner and therefore any differences in in vitro complement activation should reflect the state of the complement system in the samples. The complement C3a ELISA assay shows that the SELDI intensity reflects the serum concentration but the two measurements were not absolutely comparable. The antibody used in the ELISA recognises the C-terminus of the peptide and hence will react with any complement $\mathrm{C} 3$ cleaved at this site, whereas the SELDI peak will only report on complement C3a desarg (if, e.g., N-terminally truncated forms existed, then these would not contribute to the peak at $8940 \mathrm{~m} / \mathrm{z}$ ).

The levels of $\alpha 1$-antitrypsin determined by SELDI and Western blot (Figure 4) correlate well, indicating that $\alpha 1$-antitrypsin is elevated in the CRC patients in this study. Koomen et al (2005) recently reported that a broad SELDI peak around $51.5 \mathrm{kDa}$ was differentially detected in serum from pancreatic cancer patients compared to controls. This peak was found to contain $\alpha 1$ antitrypsin, $\alpha 1$-antichymotrypsin (as observed here) and haptoglobin. Measurement of the haptoglobin levels did not show a difference between the control and cancer patients, but as multiple proteins were found in the peak it is quite possible that the difference was owing to an altered level of one or both of the other proteins, as we show here for $\alpha 1$-antitrypsin. Like the elevation in complement $\mathrm{C} 3 \mathrm{a}$ des-arg, this suggests that an inflammatory response to the tumour is occurring. As such, it is unlikely that either protein would show high specificity for CRC (Koomen et al, 2005); however, they may be candidates for multiplexed immunoassays combining sensitive and specific biomarkers.

\section{REFERENCES}

Adam B-L, Qu YQ, Davis JW, Ward MD, Clements MA, Cazares LH, Semmes OJ, Schellhammer PF, Yasui Y, Feng Z, Wright jnr GL (2002) Serum protein fingerprinting coupled with a pattern-matching algorithm distinguishes prostate cancer from benign prostate hyperplasia and healthy men. Cancer Res 62: 3609-3614

Ahlquist DA, Skoletsky JE, Boynton KA, Harrington JJ, Mahoney DW, Pierceall WE, Thibodeau SN, Shuber AP (2000) Colorectal cancer screening by detection of altered human DNA in stool: feasibility of a multitarget assay panel. Gastroenterology 119: 1219-1227

Banez LL, Prasanna P, Sun L, Ali A, Zou Z, Adam BL, McCleod DG, Moul JW, Srivastava S (2003) Diagnostic potential of serum proteomic patterns in prostate cancer. J Urol 170: $442-446$

Bondarenko PV, Cockrill SL, Watkins LK, Cruzado ID, Macfarlane RD (1999) Mass spectral study of polymorphism of the apolipoproteins of very low density lipoprotein. JLipid Res 40: 543-555

Chen Y, Zheng S, Yu J-K, Hu X (2004) Artificial neural networks analysis of surface-enhanced laser desorption/ionisation mass spectra of serum protein pattern distinguishes colorectal cancer from healthy population. Clin Cancer Res 10: $8380-8385$

CRUK (2004) Cancer Stats - Survival. England and Wales:Cancer Research UK, www.cancerresearchuk.org

Dong SM, Traverso G, Johnson C, Geng L, Favis R, Boynton K, Hibi K, Goodman S, D'Allessio M, Paty P, Hamilton S, Sidransky D, Barany F, Levin B, Shuber A, Kinzler K, Vogelstein B, Jen J (2001) Detecting colorectal cancer in stool with the use of multiple genetic targets. $J$ Natl Cancer Inst 93: $858-865$

Fletcher RH (1986) Carcinoembryonic antigen. Ann Intern Med 104: 66-73

Greenlee R, Hill-Harmon MB, Murray T, Thun M (2001) Cancer statistics, 2001. Cancer J Clin 51: 15-36
Acute phase proteins are usually defined as proteins that change concentration by $25 \%$ or more in response to a range of inflammatory disorders. The majority of proteins increase in concentration but transferrin is one that decreases. Here, we detect an increase in a peak of approximately $79.1 \mathrm{kDa}$ in the serum of CRC patients compared to controls that was identified as transferrin (Figure 5). The primary function of transferrin is to transport iron around the body. Elevated body iron stores have been proposed to correlate with an increased risk of colon cancer and an increased proportion of transferrin loaded with iron has been linked with an increased cancer incidence, particularly in individuals who have a high intake of iron (Weinberg, 1994; Nelson, 2001; Mainous et al, 2005). It is not clear why the transferrin concentration is increased in the serum of colon cancer patients as the predicted response to inflammation is a decrease for this protein. Clearly, the cancer process is more complex than inflammation alone, and as iron appears to play a role(s) in cancer biology, it is possible that the increase in transferrin concentration observed in the serum of CRC patients is not an acute phase response.

In conclusion, proteomic profiling of serum from CRC patients and noncancer individuals, combined with the use of ANNs, can diagnose CRC with $94 \%$ sensitivity and $96 \%$ specificity in our cohort of patients. We have identified four proteins underlying six of the SELDI peaks that are significantly different between the noncancer controls and CRC patients. The proteins identified are common serum proteins and changes in their concentrations most likely reflect epiphenomena rather than secretion by cancer cells. Nonetheless, ANNs trained with just the SELDI peaks from these proteins are remarkably good at discriminating CRC, outperforming CEA.

\section{ACKNOWLEDGEMENTS}

This work was funded by Cancer Research UK.
Guang Z, Chun-Fang G, Guo-Ying S, Dong-Hui L, Xiu-Li W (2004) Identification of colorectal cancer using proteomic patters in serum. Chin J Cancer 23: 614-618

Hardcastle JD, Thomas WM, Chamberlain J, Pye G, Sheffield J, James P Balfour T, Amar S, Armitage N, Moss S (1989) Randomised, controlled trial of faecal occult blood screening for colorectal cancer. Results for first 107349 subjects. Lancet 1: $1160-1164$

Jackman RJ, Mayo CW (1951) The adenoma-carcinoma sequence in cancer of the colon. Surg Gynecol Obstet 93: 327-330

Koomen JM, Shih LN, Coombes KR, Li D, Xiao L-C, Fidler IJ, Abbruzzese JL, Kobayashi R (2005) Plasma protein profiling for diagnosis of pancreatic cancer reveals the presence of host response proteins. Clin Cancer Res 11: 1110-1118

Kozak KR, Amneua MW, Pusey SM, AL E (2003) Identification of biomarkers for ovarian cancer using strong anion-exchange proteinchips: potential use in diagnosis and prognosis. Proc Natl Acad Sci USA 100: 12343 - 12348

Kronberg O, Fenger C, Olsen J, Jorgensen OD, Sondergaard O (1996) Randomised study of screening for colorectal cancer with faecal-occult blood test. Lancet 348: $1467-1471$

Le L, Chi K, Tyldesley S, Flibotte S, Diamond D, Kuzyk M, Sadar M (2005) Identification of serum amyloid A as a biomarker to distinguish prostate cancer patients with bone lesions. Clin Chem 51: $695-707$

Li J, Rosenzweig JM, Wang YY, Chan DW (2002) Proteomics and bioinformatics approaches for identification of serum biomarkers to detect breast cancer. Clin Chem 48: 1296-1304

Mainous AG, Gill JM, Everett CJ (2005) Transferrin saturation, dietry iron intake, and risk of cancer. Ann Family Med 3: 131-137 
Malik G, Ward MD, Gupta SK, Trosset MW, Grizzle WE, Adam B-L, Diaz JI, Semmes OJ (2005) Serum levels of an isoform of apolipoprotein A-II as a potential marker for prostate cancer. Clin Cancer Res 11: $1073-1085$

Mandel JS, Bond JH, Church TR, Snover D, Bradley G, Schuman L, Ederer F (1993) Reducing mortality from colorectal cancer by screening for fecal occult blood. Minnesota colon cancer control study. $N$ Engl J Med 328: $1365-1371$

Mollnes TE, Garred P, Bergseth G (1988) Effect of time, temperature and anticoagulants on in vitro complement activation: consequences for collection and preservation of samples to be examined for complement activation. Clin Exp Immunol 73: 484-488

Muller AD, Sonnenberg A (1995) Prevention of colorectal cancer by flexible endoscopy and polypectomy. A case control study of 32702 veterans. Ann Intern Med 123: 904 -910

Nelson RL (2001) Iron and colorectal cancer risk: human studies. Nutr Rev 59: $140-148$

Newcomb PA, Norfleet RG, Storer BE, Surawicz TS, Marcus PM (1992) Screening sigmoidoscopy and colorectal cancer mortality. J Natl Cancer Inst 84: $1572-1575$

Paradis V, Degos F, Dargere D, Pham N, Belghiti J, Degott C, Janeau J-L, Bezeaud A, Delforge D, Cubizolles M, Laurendeau I, Bedossa P (2005) Identification of a new marker of hepatocellular carcinoma by serum protein profiling of patients with chronic liver disease. Hepatology 41: $40-47$

Petricoin EF, Ardekani AM, Hitt BA, Levine P, Fusaro V, Steinberg S, Mills G, Simone C, Fishman D, Kohn E, Liotta L (2002) Use of proteomic patterns in serum to identify ovarian cancer. Lancet 359: $572-575$

Poon TCW, Chan ATC, Zee B, Ho SKW, Mok TSK, Leung TWT, Johnson PJ (2001) Application of classification tree and neural network algorithms to the identification of serological liver marker profiles for the diagnosis of hepatocellular carcinoma. Oncology 61: 275-283

Poon TCW, Yip T-T, Chan ATC, Yip C, Yip V, Mok TSK, Lee CCY, Leung TWT, Ho SKW, Johnson PJ (2003) Comprehensive proteomic profiling identifies serum proteomic signatures for detection of hepatocellular carcinoma and its subtypes. Clin Chem 49: 752-760

Qu Y, Adam BL, Yasui Y, AL E (2002) Boosted decision tree analysis of surface-enhanced laser desorption/ionization mass spectral serum profiles discriminates prostate cancer from non-cancer patients. Clin Chem 48: 1835 - 1843

Ransohoff DF (2004) Rules of evidence for cancer molecular-marker discovery and validation. Nat Rev: Cancer 4: 309-313

Selby JV, Friedman GD, Quesenberry CP, Weiss NS (1992) A case-control study of screening sigmoidoscopy and mortality from colorectal cancer. $N$ Engl J Med 326: $653-657$

Tierney RP, Ballantyne GH, Modlin IM (1990) The adenoma to carcinoma sequence. Surg Gynecol Obstet 171: 81 - 94

Vlahou A, Schellhammer PF, Mendrinos S, Patel K, Kondylis F, Gong L, Nasim S, Wright GJ (2001) Development of a novel proteomic approach for the detection of transitional cell carcinoma of the bladder in urine. Am J Pathol 158: 1491 - 1502

Ward DG, Chen YC, N'Kontchou G, Thar TT, Barget N, Wei W, Billingham LJ, Martin A, Beaugrand M, Johnson P (2006) HCC induced changes in the serum proteome of hepatitis $\mathrm{C}$ infected chronic liver disease patients. Br J Cancer 94(2): $287-292$

Weinberg ED (1994) Association of iron with colorectal cancer. Biometals 7: $211-216$

Yu J-K, Chen Y-D, Zheng S (2004) An integrated approach to the detection of colorectal cancer utilizing proteomics and bioinformatics. World $J$ Gastroenterol 10: 3127 -3131 